\title{
High-pressure studies of single-molecule magnets
}

\author{
Andreas Munch Thiel, Marie Steenberg Norre, Jacob Overgaard
}

Dept. of Chemistry, Aarhus University, Langelandsgade 140,8000 Aarhus C, Denmark, andreasmt@chem.au.dk

Single-molecule magnets (SMMs) are molecular compounds possessing a magnetic bistability of their ground state, allowing them to maintain the direction of induced magnetization for a significant amount of time, after having first applied an external magnetic field [1]. Understanding the driving force behind good single-molecule magnet properties and developing improved rational synthesis design of them go hand in hand. This has been demonstrated in recent years, with record-breaking magnetic properties found in SMMs that utilizes a single Dy(III) centre in a highly axial ligand field [2-3]. A compound designed with this in mind is the pentacoordinate [Dy(Mes*O $\left.)_{2}(\mathrm{THF})_{2} \mathrm{Br}\right] 3 \mathrm{THF}$ (Mes*: 2,4,6-tri-tert-buylphenyl, THF: Tetrahydrofuran, DyBrTHF), Figure 1 (left). In a recent study on this compound, the molecular environment was found to be critical for the magnetic properties [4].

One way of systematically changing the molecular environment is through induced hydrostatic pressure. The resulting structural changes can then be probed using X-ray diffraction (XRD), by utilizing a diamond-anvil cell (DAC). We have performed high-pressure single-crystal XRD at several pressure points up until 2.9(2) GPa, and analysed the ensuing structures. Looking at the first coordination sphere, we can investigate how the applied pressure alters the molecular environment of Dy, Figure 1 (middle). At the last two pressure points, a slight drop is noted for some of the Dy-O bonds.

The magnetic properties of SMMs are closely tied to their electronic structure, which can change when undergoing external pressure, as investigated earlier in our group [5]. This information can be accessed through theoretical ab initio calculations, done here using CASSCF+NEVPT2 in ORCA. The found NEVPT2 energies of the Kramers doublets at varying pressure reveal a significant change in the energy levels, Figure 1 (right), perhaps due to the pressure-induced alteration of the ligand field.
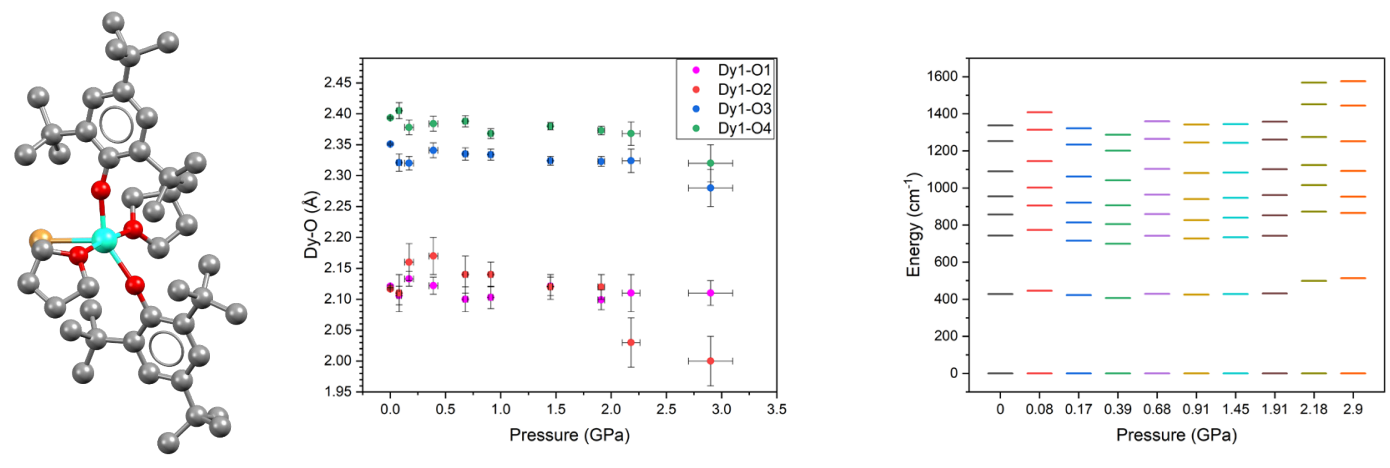

Figure 1. Left: Molecular structure of DyBrTHF. Atoms are Dy (turquoise), Br (orange), $\mathrm{O}$ (red) and C (gray). Hydrogens have been omitted. Middle: Dy-O bond-lengths of DyBrTHF with pressure. Right: NEVPT2 orbital energies of DyBrTHF as a function of pressure. Each line represents a set of degenerate Kramers doublet.

[1] Sessoli, R., et al., Magnetic bistability in a metal-ion cluster. Nature, 1993. 365, 6442

[2] Goodwin, C.A.P., et al., Molecular magnetic hysteresis at 60 kelvin in dysprosocenium. Nature, 2017. 548

[3] Guo, F.-S., et al., A Dysprosium Metallocene Single-Molecule Magnet Functioning at the Axial Limit. Angewandte Chemie International Edition, 2017. 56, 38

[4] Parmar, V.S., et al., Probing Relaxation Dynamics in Five-Coordinate Dysprosium Single-Molecule Magnets. Chemistry - A European Journal, 2020. 26, 35

[5] Thiel, A.M., E. Damgaard-Møller, and J. Overgaard, High-Pressure Crystallography as a Guide in the Design of Single-Molecule Magnets. Inorganic Chemistry, 2020. 59, 3

\section{Keywords: single-crystal diffraction; high-pressure crystallography; single-molecule magnets}

Thanks to the David Mills group, University of Manchester for providing me with crystals of the type DyBrTHF.

Acta Cryst. (2021), A77, C349 